\title{
Effect of Frequency Selective Shield of Semielliptical Shape on the Characteristics of Antenna Array
}

\author{
D.V. Semenikhina,A.I. Semenikhin, Y.V. Yukhanov \\ Department of Antennas and Radio Transmitters \\ Southern Federal University \\ Southern Federal,Russia
}

\begin{abstract}
Characteristics of a rectangular waveguide array with a frequency-selective shield of semielliptical shape with expansion of an operating band are investigated. It is shown that the region of transparency of the shield displaces down in a frequency in a comparison with the plane infinite frequency-selective surface (FSS). In this region the shield slightly changes the form of the main lobe of radiation pattern of an antenna array and increases far-out side lobes. Out of this region the shield reduces a backward specular scattering from an antenna, but increases a bistatic scattering.
\end{abstract}

Keywords-antenna array; frequency-selective surface; shield; radiation pattern; scattering characteristic

\section{INTRODUCTION}

Frequency selective surfaces (FSS) as shields are widely used to solve various problems of electromagnetic compatibility (EMC) and radar cross-section (RCS) reduction [1-4]. These shields are set in front of the antenna arrays and they are used to reflect all out-of-band signals away from the antenna array while allowing in-band signals to reach the antenna array [3]. A review of the open literature shows that not much information has been published on the RCS of antenna arrays. The work [3] discusses some of the fundamental considerations in providing low RCS of an antenna. Usually the use of a shaped FSS in front of an antenna makes the RCS reduction task no a wideband and narrowband problem.

In connection with this a shield bandwidth expansion is an actual problem.

The creation of FSS-shield includes design of topology of a single cell of FSS, an analysis of radiation and scattering characteristics of antenna array with the shield and a research of a configuration of an entire shield taking into account EMC requirements. The shield should provide the small return losses and required radiation characteristics of antenna array at operating frequencies and necessary EMC level in the required angular sector out of this band.

With a help of an accurate method for the computation of the RCS of active waveguide arrays it is shown [4] that backscattering pattern (BSP) and bistatic scattering pattern of waveguide antenna array (without FSS-shield) are essentially dependent on the frequency. At operating frequency band the level of BSP main lobe is on $9-10 \mathrm{~dB}$ less, than for the equivalent metal plate [3]. At low (non-operating) frequencies the waveguides is evanescent waveguides; secondary field grows and diffraction lobes are absent. At high (non-operating) frequencies the diffraction lobe level increases and may exceed the level of the BSP main lobe. An effect of radomes especially in the form of FSS-shield in scattered fields for antennas and phased array antennas is less studied. To research it, approximate approaches are generally used.

In [4] on the basis of system of integral equations the numerical method is constructed and scattering fields of two-dimensional model of a two-reflector antenna under the peaked dielectric radome are calculated. A backscattering from three-dimensional models of a reflector antenna under the tapered dielectric radome is approximately estimated. Importance of taking into account of all electromagnetic interactions in "antenna-radome" system [4] is emphasized.

One of the possible shield types was discussed in authors' paper [5].

The aim of this paper is to search the way of frequency band expansion of the waveguide array with the polarizable-independent FSS-shield of the semielliptical form. Simulation of characteristics of "array-FSS" system was executed using Ansoft HFSS package.

\section{MODELS OF FSS AND ANTENNA ARRAY}

\section{A. Characteristics of A Plane Model of \\ Polarizable-Independent FSS}

The simple model of researched FSS was based on the theory of infinite periodical structures. The main feature of the model is high density of element packaging. Each element is mutually perpendicular modified $\mathrm{H}$-shaped slots (fig.1 (a)). They are formed between perfectly thin tape conductors of 0.5 mm width.

The lattice has much more complex structure of slots in comparison with published earlier (fig.1 (b)) [5].

The cell of one-layer FSS has sizes of $12.27 \mathrm{~mm} \times 12.27$ $\mathrm{mm}$.

The frequency responses of transmission coefficient $|\mathrm{T}|$ for different angles of incidence $\theta=0^{\circ}-60^{\circ}$ on FSS in H-plane are illustrated by fig.2. As seen, a return losses in case of normal wave incidence do not exceed $0.5 \mathrm{~dB}$ in the band of $8.5 \mathrm{GHz}$ to $11.8 \mathrm{GHz}$ (fig 2 (a)). The working bandwidth is $200 \mathrm{MHz}$ more, than in the case considered earlier [5] (fig 2 (b)). In the sector of incidence angles $\pm 60^{\circ}$ the losses in a band of $8.2-9.65 \mathrm{GHz}$ are less, than $0.8 \mathrm{~dB}$. 


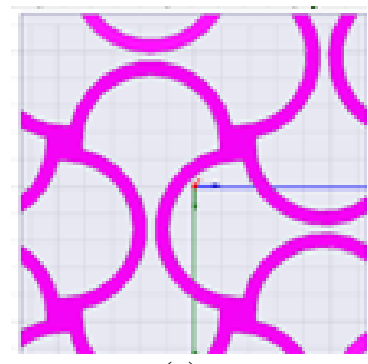

(a)

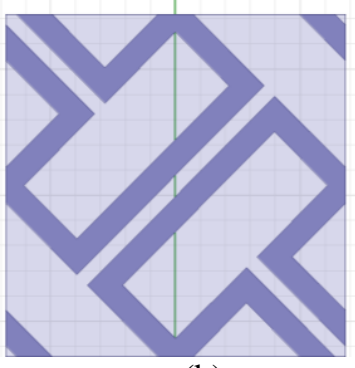

(b)
FIGURE I. (A) CELLS OF MODIFIED H-SHAPED SLOTS AND (B) H-SHAPED SLOTS

Below this FSS model was used for design of polarizable-independent passband shield of antenna array.

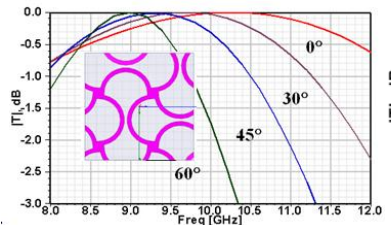

(a)

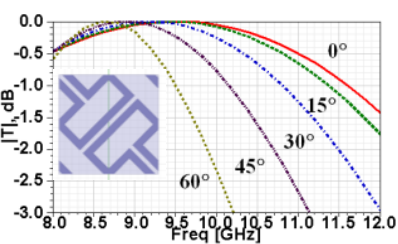

(b)
FIGURE II. (A) TRANSMISSION COEFFICIENTS OF INFINITE PLANE FOR LATTICES OF MODIFIED H-SHAPED SLOTS AND (B) H-SHAPED SLOTS

\section{B. Model of the Waveguide Antenna Array Covered by FSS-Shield}

The antenna curtain $(886 \mathrm{~mm} \times 1145 \mathrm{~mm}$ sizes $)$ consists of a lattice of rectangular waveguide open ends in a metal base. The configuration of the FSS-shield was set in a form of a cylindrical surface of semielliptical form with $946 \mathrm{~mm}$ major axis and $473 \mathrm{~mm}$ semiminor axis (fig. 3).

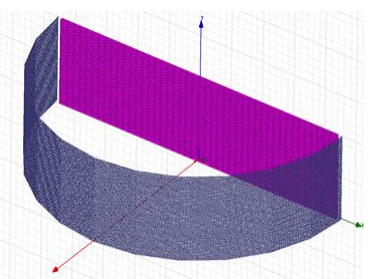

FIGURE III. RECTANGULAR WAVEGUIDE ANTENNA ARRAY WITH FSS-SHIELD

In E-plane (on Y-axis) periodic boundary conditions with the period equal to the period of FSS were used (fig. 4).

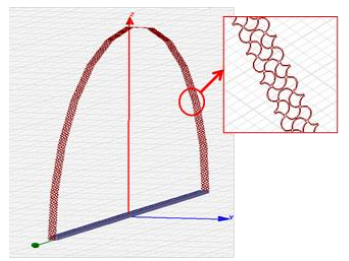

FIGURE IV. UNIT CELL OF FSS-SHIELD WITH MODIFIED H-SHAPED SLOTS AND ANTENNA ARRAY

Thus, the computer model of FSS-shield system includes a finite number of waveguide open ends with ports in the metal base (fig. 5 (a)) and finite set of FSS cells on plane chamfers of the shield in horizontal H-plane (fig. 5 (b)).

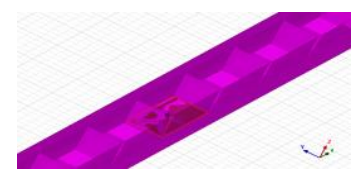

(a)

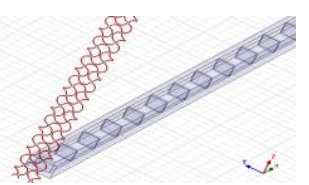

(b)
FIGURE V. (A) THE WAVEGUIDE ANTENNA ARRAY CELL WITH PORTS IN THE METAL BASE AND (B) THE CELL OF THE SHIELD IN HORIZONTAL H-PLANE

There are defects of topology of FSS in form of closed rings on joints of adjacent faces (near YOZ-plane, fig. 6). In vertical E-plane also the finite number of FSS cells is had in one Floquet's period of the waveguide lattice.

Calculation precision after 11th steps, when the construction was divided on 877787 tetrahedra, was 0.034 .

Characteristics of the system of the finite size in vertical direction were calculated without taking into account edge effects.

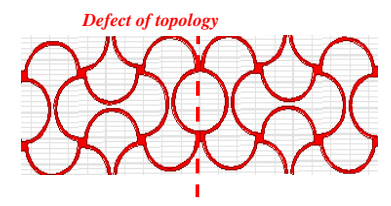

FIGURE VI. JOINT OF ADJACENT FACES NEAR THE YOZ PLANE

\section{NUMERICAL RESULTS}

\section{A. Radiation Characteristics of Antenna Array with}

\section{FSS-Shield}

Fig.7 illustrates frequency responses of the maximum realized gains of inphase uniform-amplitude AA with the shield and without shield. The level of diffraction losses because of the shield is $0.5 \mathrm{~dB}$ in band of $8.6-9.1 \mathrm{GHz}$. It is more, than a band of FSS-shield developed by earlier [5]. The losses increase to $0.7 \mathrm{~dB}$ at lower frequencies $(8-8.6 \mathrm{GHz})$ and to $2.4 \mathrm{~dB}$ at higher frequencies $(9.1-12 \mathrm{GHz})$.

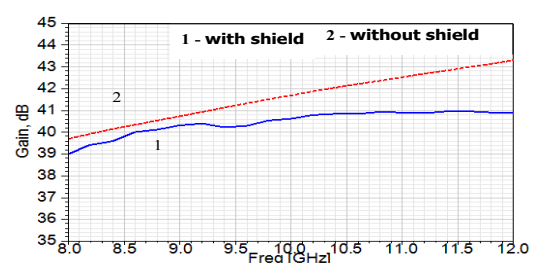

FIGURE VII. REALIZED GAINS OF INPHASE UNIFORM-AMPLITUDE ANTENNA ARRAY WITH THE SHIELD AND WITHOUT SHIELD

The region of transparency of semielliptical shield is shifted down along frequency axis as for the model of an infinite planar FSS for $\theta=60^{\circ}$.

In horizontal $\mathrm{H}$-plane in the bandpass range the shield 
influences the radiation pattern shape weakly (fig. 8).

The null beamwidth increases approximately on $0.2^{\circ}$ (without shield $-4.2^{\circ}$ ). Because of the radiation of secondary currents on a convex side surface of the shield, distant side lobes of radiation pattern increase by $5-6 \mathrm{~dB}$.

The interference of fields in FSS-shield system not only changes FSS frequency response but is especially noticeable near side and two central chamfers of the shield with defects of FSS topology near to a front edges of the shield.

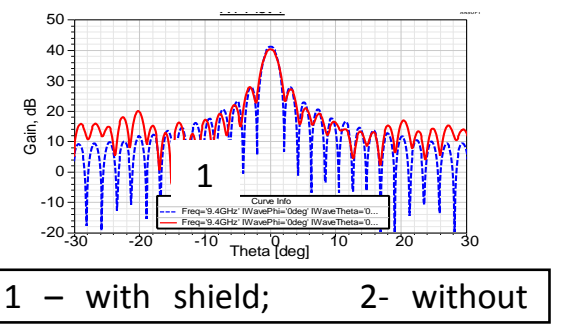

FIGURE VIII. RADIATION PATTERNS OF INPHASE

UNIFORM-AMPLITUDE ANTENNA ARRAY WITH THE SHIELD AND WITHOUT SHIELD

\section{B. Scattering Characteristics of Antenna Array with FSS-Shield}

In fig. 9 the frequency dependences of backscattered fields of inphase uniform-amplitude antenna array are given for the linear working polarization in case of normal incidence (along a normal to an aperture).
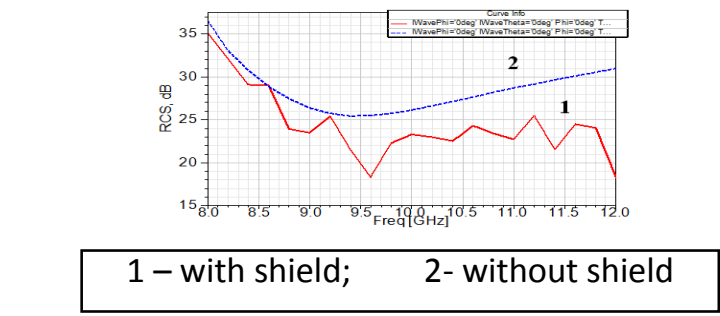

FIGURE IX. BACKSCATTERED FIELDS OF INPHASE UNIFORM-AMPLITUDE ANTENNA ARRAY WITH THE SHIELD AND WITHOUT SHIELD

As seen in Fig.9, the level of backscattered field of antenna array with the shield (curve 1) is less, than without the shield (curve 2) on $1-2 \mathrm{~dB}$ in a band of $8-8.6 \mathrm{GHz}$ and on $3-12$ $\mathrm{dB}$ - in a band of $9.35-12 \mathrm{GHz}$.

The intensive side lobes caused by diffraction on chamfers of the shield at the edge of its working band (where its reflecting action is great) are watched in scattering diagram (fig. 10).

The angular regions with the raised scattering are seen. It is the beam reflected from an antenna curtain in FSS passband (at frequency $8 \mathrm{GHz}$ ). Here the shield inserts the small contribution to the overall scattering field. With growth of frequency up to $12 \mathrm{GHz}$ the FSS screens the antenna, the scattering field in a reflection direction sharply decreases (till
$19-30 \mathrm{~dB}$ ) in comparison with a field at frequency $8 \mathrm{GHz}$.

The shadow lobe is well seen. The reflected beam is weaker, than shadow lobe on $6-24 \mathrm{~dB}$ due to absorption in matched waveguide lattice. There are the characteristic side lobes in scattering diagram. They are caused by diffraction on facets of the shield on edge of its operating band where a reflecting action of the shield grows.

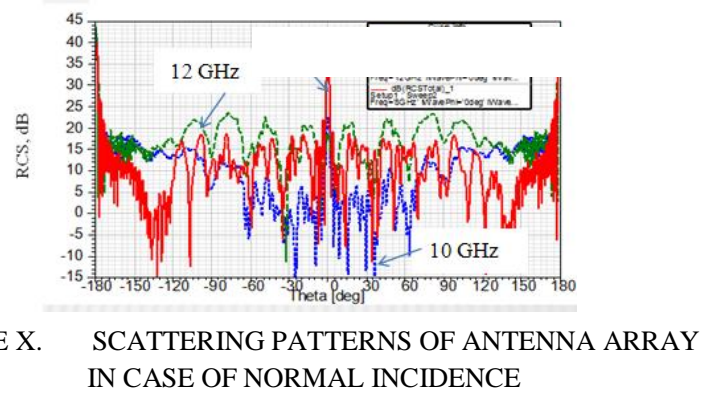

\section{CONCLUSION}

Thus, the model of polarizable-independent plane FSS with expanded band of operating frequencies and the model of waveguide antenna array covered by FSS-shield of the semielliptical form are considered.

Numerically influence of the form of the shield on characteristics of radiation and scattering of system of "array-shield" is estimated. It is shown that the region of transparency of the semielliptical shield (at level of insertion losses of $0.5 \mathrm{~dB}$ ) is in limits $8.6-9.1 \mathrm{GHz}$ and is displaced down along frequency axis in comparison with plane model of the infinite FSS. In this region the shield slightly changes the form of the main and first side lobes of radiation pattern of an antenna array and increases far-out side lobes by $5-6 \mathrm{~dB}$. Out of this region the shield in addition reduces backward specular scattering from the antenna by $3-12 \mathrm{~dB}$, but increases bistatic scattering.

\section{ACKNOWLEDGMENT}

This work was performed as project part of the state task No. 8.2461.2014/K of the Ministry of Education of Russia.

\section{REFERENCES}

[1] Lagarkov A.N. \& Pogosyan M.A., Fundamental and applied problem of Sthels-technologies, News of Russia Science Academy, vol.73, p. 848, Sep, 2003.

[2] Munk B., Finite Antenna Arrays and FSS. John Wiley \& Sons, Hoboken, NJ, 2003.

[3] Knott E.F. et al., Radar Cross Section. 2nd Edition, SciTech, Raleigh, NC, 2004.

[4] Chan K.K. \& Chadwick K., Computation of Radar Cross Section of Waveguide Arrays, Anten. and Propag. Society Intern. Symp. V.3. pp. 1746-1749, Aug 1999.

[5] YukhanovY.V., Semenikhin A.I. \& Semenikhina D.V., Modeling of the characteristics of the waveguide phased array with frequency-selective shield, IX International Conference on Antenna Theory and Techniques (ICATT), pp. 241-243, 2013. 